\title{
$\$$ Research Square

\section{Effects of Infections of Helicobacter Pylori With Different Virulent Factors on Severity of Gastrointestinal Diseases}

\author{
Yan Zhang \\ Wuhan University of Science and Technology \\ Xiang-ming Fang \\ Wuhan University of Science and Technology \\ Kui Tian ( $\nabla$ trrkui0918@163.com ) \\ Wuhan Pulmonary Hosptial https://orcid.org/0000-0002-4330-849X
}

\section{Research article}

Keywords: Helicobacter Pylori infection, Antibody types, Gastrointestinal diseases, Endoscopy, Pathology, CagA/VacA

Posted Date: January 19th, 2021

DOI: https://doi.org/10.21203/rs.3.rs-148511/v1

License: (c) (i) This work is licensed under a Creative Commons Attribution 4.0 International License. Read Full License 


\section{Abstract}

Background: Helicobacter Pylori (H. pylori) infection, one of the most common

chronic bacterial infections, has been considered as a major cause of diseases such as lymphoma, gastritis, peptic ulcers, and stomach cancer. Here, we aimed to determine whether $H$. pylori strains with different virulence contribute to the gastrointestinal diseases differentially in clinical settings, which may provide future direction for eradication of $H$. pylori infection.

Methods: We recruited 501 patients with gastrointestinal disorders for analysis of antibody types of $H$. pylori infection. Correlation analysis was done to determine the association of different virulence of $H$. pylori with patients' baseline parameters and personal disease history. Next, subjects with each type of anti- H. pylori infection antibody were subjected to esophagogastro duodenoscopy $\mathbb{E G D}$ ) and colonoscopy examinations. The pathological diagnosis was also conducted in endoscopic samples. Chi-squared test was employed to compare the differences in endoscopic assessments and pathological findings among three types of $H$. pylori infection determined by the presence of antibodies to virulent factors.

Results: There were 296 cases with Type I H. pylori infection, 120 cases with Type II H. pylori infection, and 85 cases without $H$. pylori infection (negative, Type III). No correlation was found between different virulence of $H$. pylori and participants' baseline data $(P>0.05)$. EGD results showed that the incidences of peptic ulcer, bleeding and malignant lesions in Type I group were significantly higher than that in Type II and Type III (Pख0.05). Despite of increased trends of incidences of precancerous alterations and the malignance in Type I group compared with type II and III groups, there was no significant difference $(P>0.05)$. In addition, coloscopic features were similar among three groups. On the other hand, infections of $H$. pylori with cytotoxin-associated gene A (CagA) and/or vacuolating cytotoxin $A(\operatorname{Vac} A)$ virulent factors resulted in more severe histopathological diseases than that with only Ure $A / B$ factor and without infection $(P<0.05)$.

Conclusions: Infections of $H$. pylori strains with CagA/VacA are likely to cause development of severe gastrointestinal diseases. These results are helpful to treat for $H$. pylori infection clinically.

\section{Background}

Helicobacter Pylori (H. pylori) infection contributes to the development of chronic gastritis, peptic ulcer, and even gastric cancer and gastric Mucosa-Associated Lymphoid Tissue lymphoma (MALT) ${ }^{[1,2]}$. H. pylori has been declared and ratified as carcinogen I by the International Agency for Research on Cancer (IARC), which is part of the World Health Organization (WHO). In addition to diseases in its primary site of infection, a growing evidence demonstrates that $H$. pylori infection is involved in intestinal diseases, idiopathic thrombocytopenic purpura disease, iron deficiency anemia disease, coronary heart disease, infertility and other pathogeneses ${ }^{[3]}$. Therefore, accurate diagnosis and proper treatment of $H$. pylori infection are crucial to the progress and prognosis of clinical patients.

So far, H. pylori is determined using a variety of methods including urea breath test (UBT), serological test, stool antigen test, histological culture, etc.. Although UBT has been considered as a preferred non-invasive examination for diagnosis of $H$. pylori infection, many factors affect the accuracy of UBT, and lead to false negative and positive results. Besides that, UBT fails to categorize $H$. pylori into highly- and low-virulent strains based on the presence or absence of two key virulence factors, cytotoxin-associated gene $A(\operatorname{Cag} A)$ and/or vacuolating cytotoxin $A(\operatorname{Vac} A)^{[4]}$. 
In clinical practices, patients are often requested to eradicate $H$. pylori infection if the subject is tested positive using UBT. According to the 2019 Expert Consensus Opinion on Eradication, Prevention and Control of Gastric Cancer in Helicobacter Pylori China (Shanghai, 2019), the elimination of $H$. pylori helps to control the outcome of gastric cancer ${ }^{[5]}$. However, the role of different virulence of $H$. pylori in the development of gastrointestinal diseases remains unknown. Until now, based on antibodies against the bacterial virulence factors in serum, patients with $H$. pylori infection are classified into three types in accordance with the presence of CagA, VacA and Urease A/B (Ure A/B) or absence. We hypothesized that different virulent factors predict the severity and outcomes of gastrointestinal diseases in those patients with H. pylori infection. In this study, serum samples from 501 patients who had been hospitalized for gastrointestinal symptoms from September 2019 to June 2020 in the Department of gastroenterology at Puren Hospital in Wuhan were collected for evaluation of bacterial virulence. By doing so, we anticipate to solve the puzzle about the effects of different virulent factors of with $H$. pylori on gastrointestinal diseases.

\section{Methods}

\section{Patients}

We recruited consecutively 501 patients with gastrointestinal symptoms (abdominal pain, abdominal distension, acid reflux, etc.) in the Department of Gastroenterology at Puren Hospital from September 2019 to May 2020 for $H$. pylori antibody tests and other examinations. All patients were informed consent, including 260 males and 241 females, with a minimum age of 15 years and a maximum age of 90 years (average age of $57.02 \pm 14.01$ ). The baseline data of the patients included sex, age, smoking history, alcohol consumption history and family history of cancers.

Exclusion criteria: Patients with severe cardiovascular, hepatic, kidney dysfunction, immune deficiency disease, those with esophagogastric variceal hemorrhage, pregnant women or with mental illness, previous anti- $H$. pylori administration or those undergoing $H$. pylori eradication treatment were also excluded.

All subjects were briefed about this study, and had signed the informed consent form before the enrollment. The study protocol was also approved by the institutional review board (IRB) at the Puren Hospital.

Measurements of types of virulent factors of $H$. pylori infection

Types of $H$. pylori antibody in the patient serum were determined using a detection kit produced by Shenzhen Brautt Company, and Western blotting method. The manufacturer's instructions were followed. The kit classified the infections as Type I, Type II and Type III (negative) based on three types of antibody scenarios. Type I is positive for CagA and/or VacA antibody and Ure A/B antibody. Type II is positive for Ure A/B antibody, and negative for CagA and/or VacA antibody. Type III (Negative) is negative for CagA, VacA, and Ure A/B antibodies.

\section{Esophagogastroduodenoscopy and colonoscopy}

Of the 501 patients who underwent the antibody classification measurement, 384 patients received esophagogastroduodenoscopy (EGD) and 136 patients received colonoscopy. All participants signed the informed consent for their respective examinations. To those who would receive endoscopic therapy (such as: hemostasis with gastric and duodenal ulcer, excision of polyps), the informed consent for those operations were also obtained. EGD and colonoscopy were performed using the Olympus GIF-HQ290 and Olympus CF-H290L / I, respectively. 
Endoscopists with more than 5 years of experience reviewed the endoscopic images and then made final endoscopic diagnosis.

Pathological examination

Except bearing the risk of hemorrhage in certain sorts of lesion locations by sampling, lesions of all patients who underwent endoscopy were collected by endoscopists and then were diagnosed by pathologists. The slides were reviewed by three pathologists who have worked for more than 5 years. If two pathologists disagreed with the diagnosis, the third pathologist then reviewed the slides to reach a consensus.

\section{Statistical Analysis}

First, SPSS 23.0 software was used to perform correlation analysis to determine the association between different antibody types of $H$. pylori infection and age, sex, smoking, alcohol consumption and family history of cancer according to the baseline data of 501 patients involved. Secondly, we conducted the Chi-squared test to compare the effects of presence or absence of antibodies to the virulent factors of $\mathrm{H}$. pylori in those patients on gastrointestinal diseases via endoscopy and pathological measurements. A two-sided $P$-value less than 0.05 was considered statistically significant.

\section{Results}

H. pylori antibody types were not correlated with age, sex and personal history

Among 501 patients examined for the antibodies to the virulent factor of $H$. pylori infection, 296 cases were classified as Type I H. pylori infection, 120 cases were classified as Type II and 85 cases were classified as Type III (negative) $H$. pylori infection. Correlation analysis indicated that none of three groups (Type I, Type II and negative) showed any significant association with patients' baseline data (age, sex), and risk factors (e. g. smoking, alcohol consumption, family history of cancer) $(P>0.05)$ as shown in Table 1.

Table 1 Correlation analysis between different virulence of $\boldsymbol{H}$. pylori infection and baseline parameters

\begin{tabular}{|c|c|c|c|c|c|}
\hline Factors & Type I & Type II & Type III & Coefficient & $\begin{array}{l}P \\
\text { value }\end{array}$ \\
\hline Age & $57.35 \pm 14.02$ & $57.78 \pm 13.30$ & $54.78 \pm 14.89$ & 0.05 & 0.229 \\
\hline Female/male & $135(45.6 \%) / 161(54.4 \%)$ & $59(49.2 \% / 61(50.8 \%)$ & $47(55.3 \%) / 38(44.7 \%)$ & 0.07 & 0.09 \\
\hline smoking & $81 \rrbracket 27.2 \% \bigotimes$ & $25 \rrbracket 20.8 \% \rrbracket$ & $15 \rrbracket 17.6 \% \rrbracket$ & 0.08 & 0.073 \\
\hline alcohol & $46 \rrbracket 15.5 \% \bigotimes$ & $12 \bigotimes 10 \% \rrbracket$ & $10 \otimes 11.8 \% \rrbracket$ & 0.04 & 0.393 \\
\hline $\begin{array}{l}\text { Cancer } \\
\text { history }\end{array}$ & $18 \rrbracket 6.1 \% \square$ & $6 \rrbracket 5 \% \bigotimes$ & $3 \rrbracket 3.5 \% \rrbracket$ & 0.05 & 0.231 \\
\hline
\end{tabular}

\section{Gastroscopic features of three groups based on antibody detection}


Out of 501 subjects, 384 patients received EGD examination. In them, we found 234 (79.1\%) in type I group, 86 (71.7\%) in type II, and $64(75.3 \%)$ in the negative group. After statistical analysis, the percentage numbers of patients receiving gastroscopic examinations were not different among three types $(P>0.05)$. As shown in Table 2 , in type I group, we found 47 cases of chronic gastritis (20.1\%) confirmed by EGD, 19 cases with reflux esophagitis (RE, 8.1\%), 91 cases with peptic ulcer with hemorrhage (38.9\%), 62 cases with polyps in esophagus, gastric or duodenum (26.5\%), 15 cases with gastric malignancies (6.4\%). Of the 86 cases in type II, we found 15 cases (17.4\%) with chronic gastritis, 20 cases (23.3\%) with esophagitis, 17 cases (19.8\%) with peptic ulcer with hemorrhage, 33 cases (38.4\%) with polyps in esophagus, gastric and duodenum, and only 1 case (1.2\%) with gastric malignancies. In the negative group, we found 17 cases $(26.6 \%)$ with chronic gastritis, 9 cases with $(14.1 \%)$ reflux esophagitis, 8 cases (12.5\%) with peptic ulcer with hemorrhage, 28 cases (43.8\%) with polyps in esophagus, stomach or duodenum, and 2 cases (3.1\%) with possible gastric malignant change. As shown in Table 2, the primary gastroscopic characteristics of type I group were gastric and duodenal ulcers with or without hemorrhage, which had a total rate of $38.9 \%$. This number in type I group was significantly higher than that in type II and negative groups $(P<0.05)$. More interestingly, the ratio of neoplasia findings in type I group was also significantly higher than that in the other two groups $(P<0.05)$. On the other hand, the combined rate of polyps in esophagus, stomach and duodenum was comparatively added in type II and negative group, which accompanied with reduction of incidence of ulcers, bleeding and neoplasia findings.

Table 2

Gastroscopic features with different virulence factors of $H$. pylori

\begin{tabular}{|c|c|c|c|c|c|}
\hline Type & Gastritis & $\mathrm{RE}$ & Peptic ulcer/ Bleeding & Polyps & Cancer \\
\hline । & $47(20.1 \%)$ & $19(8.1 \%)$ & $91(38.9 \%)^{\star}$ & $62(26.5 \%)$ & $15(6.4 \%)^{*}$ \\
\hline II & $15(17.4 \%)$ & $20(23.3 \%)$ & 17(19.8\%) & $33(38.4 \%)$ & $1(1.2 \%)$ \\
\hline III & $17(26.6 \%)$ & $9(14.1 \%)$ & $8(12.5 \%)$ & $28(43.8 \%)$ & $2(3.1 \%)$ \\
\hline
\end{tabular}

Pathological results with different virulence factors of $H$. pylori

Exclusion of high risk in hemorrhage, 294 lesions were sampled to identify pathological alterations in patients of the three types. As shown in Table 3, these included 173 cases (73.9\%) in type I group, 72 cases (83.7\%) in type II group, and 49 cases $(76.6 \%)$ in negative group. There was no significant difference in rates of patients with pathological changes $(P>0.05)$. Firstly, we divided pathological results into three phenotypes comprising mucosal atrophy and/or intestinal metaplasia, intraepithelial Neoplasia/gastric cancer, and other benign pathological findings. As was shown in Table 3, there were 41 (23.7\%) cases with atrophy /intestinal metaplasia, 17 (9.8\%) cases with intraepithelial neoplasia and carcinoma, and 115 (66.5\%) with benign lesions in type I group. Of the 72 patients in type II group, there were $14(19.4 \%)$ cases with atrophy and/or intestinal metaplasia, 3 (4.2\%) cases with intraepithelial neoplasia/carcinoma, and 55 (76.4\%) cases with diagnosed benign lesions. Of the 49 negative cases, there were 9 $(18.4 \%)$ cases with atrophy and/or intestinal metaplasia, 2 (4.1\%) cases with intraepithelial neoplasia/carcinoma, and $38(77.6 \%)$ cases with other benign lesions. Statistical analysis indicated that the incidences of precancerous alterations and the malignance in type I group were elevated in comparison with that in type II and negative groups. There was no significant difference of any of these parameters among three groups as shown in Table $3(P>0.05)$. 
Table 3

Pathological manifestations with different types of $H$. pylori infection

\begin{tabular}{|lllll|}
\hline Type & Atrophy/Metaplasia & Neoplasia/Cancer & Benign & Total \\
\hline I & $41(23.7 \%)$ & $17(9.8 \%)$ & $115(66.6 \%)$ & $173(100 \%)$ \\
\hline II & $14(19.4 \%)$ & $3(4.2 \%)$ & $55(76.4 \%)$ & $72(100 \%)$ \\
\hline III & $9(18.4 \%)$ & $2(4.1 \%)$ & $38(77.6 \%)$ & $49(100 \%)$ \\
\hline
\end{tabular}

Colonoscopy features in $\mathrm{H}$. pylori positive patients with different virulent factors

Of 501 patients involved in antibody classification, 136 cases received the colonoscopy examination, which included $72(24.3 \%)$ cases in type I group, $40(33.3 \%)$ cases in type II group and $24(28.2 \%)$ subjects in the negative group.

The percentage numbers of examined cases among three type groups are not different $(P>0.05)$. As shown in Table 4, $46(63.9 \%)$ cases in type I group had polyps, adenomatous polyps and cancer. In type I patients, $6(8.3 \%)$ cases had inflammatory alteration and inflammatory bowel disease (IBD), and $20(27.8 \%)$ cases appeared to be normal under the colonoscopy examination. Among the 40 subjects in type II group, 23 cases (57.5\%) had polyps, adenomatous polyps and malignance endoscopic features. There were 3 cases $(7.5 \%)$ with inflammatory alteration and IBD, while 14 cases (25\%) had normal colonoscopy results. Lastly, 15 patients out of $24(62.5 \%)$ examined had polyps, adenomas and malignant morphologic types in the negative group. There were 3 cases $(12.5 \%)$ with inflammatory alterations and IBD, and 6 cases $(25 \%)$ with normal colonoscopy results. Results of Chi-square test indicated that colonoscopy results were not significantly different among three groups $(P>0.05)$.

Table 4

Colonoscopic findings with different types of $H$. pylori infection

\begin{tabular}{|lllll|}
\hline Type & Polyps/Adenoma/Cancer & Inflammation & Normal & Total \\
\hline I & $46(63.9 \%)$ & $6(8.3 \%)$ & $20(27.8 \%)$ & $72(100 \%)$ \\
\hline II & $23(57.5 \%)$ & $3(7.5 \%)$ & $14(35 \%)$ & $40(100 \%)$ \\
\hline
\end{tabular}

Histopathology difference of colonoscopy results from patients in different virulence factor groups

Tissue samples from 55 subjects out of the 130 patients receiving colonoscopy examination were evaluated for pathological changes. As shown in Fig. 1, 10 (38.5\%) out of 26 cases in type I group were diagnosed pathologically as mild to moderate inflammation and hyperplastic polyp. The remaining 16 cases $(61.5 \%)$ were considered to have severe inflammation, adenomatous polyps, atypical hyperplasia and cancer. In type II group, 14 (70\%) out of the 20 cases had mild and moderate inflammation, while the remaining 6 cases $(30 \%)$ had severe inflammation, and adenomatous polyps. Of 9 subjects in the negative group, $7(77.8 \%)$ cases were diagnosed as mild to moderate inflammation, and hyperplastic polyps, while the remaining 2 cases $(22.2 \%)$ had severe inflammation phenotypes. Statistical analysis demonstrated that infection of $H$. pylori with CagA and/or VacA resulted in significantly more severe histopathological alterations in comparison with that with only Ure A/B virulent factor or without infection $(P$ $<0.05$ ).

\section{Discussion}


H. pylori is a group of gram-negative spirochetes widely colonized in the stomach. More than $50 \%$ of the world's population is currently infected with the bacteria, which have been identified by the International Cancer

Organization as the leading cause of gastroduodenal ulcers, chronic gastritis and stomach cancer ${ }^{[6]}$. The genome of $H$. pylori has high genetic diversity which affects its invasion to the gastric mucosa of the host. The mechanism of its pathogenicity is related to the interactions among virulence factors, host genes and environmental factors. The genome of $H$. pylori encodes more than 1,500 proteins, of which more than 500 genes are specific for $H$. pylori. The pathogenic ability of $H$. pylori strains is diverse, so the development and progression of host diseases caused by $H$. pylori strains vary. So far, the known virulence factors of $H$. pylori strains have included CagA, VacA, Urease A/B, DupA, OMPs and ICEA, of which CagA, VacA and Urease A/B are the best characterized and investigated ones ${ }^{[7-9]}$.

Based on the presence or absence of the major three virulence factors (CagA, VacA and Urease A/B), patients with $H$. pylori infection can be divided into three types. The data presented in this manuscript are from 501 participants. We observed that the incidence of type I infection is higher than that of Type II and negative group. The correlation analysis revealed that there is no association between different virulence of $H$. pylori and patients' baseline parameters and personal history including age, sex, history of smoking, alcohol consumption, and family history of cancer. In the future, we plan to recruit more participants to further evaluate whether any of the risk factors of hypertension, diabetes and hyperlipidemia is related to the different virulence of $H$. pylori infection.

It has been established that the highly virulent $H$. pylori strains comprise the cytotoxin-associated genes pathogenicity island (CagPAl), which is a $40 \mathrm{~kb}$ region containing 31 genes encoding the elements of a type IV secretion system, participating in CagA toxic activity and following a series of inflammatory responses ${ }^{[10]}$. Research has shown that infections with CagA positive strains are more virulent than the strains without this genotype in gastric colonization and proliferation ${ }^{[11]}$. In the process of infection, CagA is localized on the plasma membrane, in which it is phosphorylated at specific Glu-Pro-lle-Tyr-Ala (EPIYA)-motifs through Src and Abl kinases in host ${ }^{[12,13]}$. The degree of toxicity with CagA is associated highly with numbers and types of the EPIYA-motifs at the C-terminal region. After translocation, CagA interacts with multiple host cell molecules and induces the dysregulation involved in the homeostatic signal transduction of gastric epithelial cells, triggering chronic pro-inflammatory responses involving apoptosis, disruption of cell polarity and promotion of genetic instability, through which carcinogenesis then takes place. Owing to such cancer-inducing traits, CagA therefore has been designated as the first bacterial oncoprotein ${ }^{[14]}$.

The vacuolating cytotoxin $A(\operatorname{Vac} A)$ is known for its capacity to induce the formation of vacuoles in eukaryotic cells. VacA gene exists in all $H$. pylori strains, with different vacuolating ability, which is conferred by variations in five VacA regions: s-region ( $\mathrm{s} 1$ and s2), i-region (i1, i2, i3), m-region ( $\mathrm{m} 1$ and $\mathrm{m} 2$ ), d-region ( $\mathrm{d} 1$ and $\mathrm{d} 2$ ), and the recently identified c-region (c1 and c2). Different variants of VacA like s1, s2, m1 and others could bring about varied toxic effects from cellular vacuolation, oxidative stress to apoptosis induction ${ }^{[15,16]}$. According to results from Ogiwara $H$, the potential risk of developing peptic ulcers and cancer in patients carrying s1 or $\mathrm{m} 1$ is significantly higher than those carrying other toxins ${ }^{[17]}$. In Iran, for example, the d1 was identified as a novel oncogenic factor for gastric cancer ${ }^{[18]}$. Additionally $\mathrm{d} 1$ and $\mathrm{i} 1$ have displayed a synergistic effect on the progress of gastric cancer ${ }^{[19]}$. Once infected, VacA induces the vacuolation of epithelial cells, triggers the release of mitochondrial cytochrome $\mathrm{C}$ and then initiates apoptosis ${ }^{[20]}$. Moreover, it also confers the formation of membrane channels, through which cytochrome $\mathrm{C}$ is released and then binds to extracellular receptors. As a result, cellular inflammatory responses occur consequently [21,22]. 
In our present study, we found that the probability of peptic ulcer, gastrointestinal bleeding and malignancy increased significantly in the type I patients carrying $H$. pylori with CagA/Vaga compared with type II and negative counterparts. At the same time, infections of $H$. pylori with UreA/B show primarily as relative mild gastroscopic manifestations like gastritis, reflux esophagitis, polypoid changes. In regard to mechanism, we reason that the main virulent factors CagA and VagA act on the gastric and duodenal mucosa, and trigger a series of inflammatory reactions, which lead to relatively severe gastroscopic outcomes such as peptic ulcer, bleeding, and even cancer. UreA/B in Type II group is less virulent than CagA/VacA in type I group, which could only influence vesicular transport and cell cycles ${ }^{[23]}$. As a result, gastroscopic characteristics are based on chronic inflammatory cell infiltration of the mild to moderate inflammatory response without ulcers and malignant changes. Although precancerous changes (atrophy, intestinal metaplasia, intraepithelial neoplasia) and cancers in type I group show a trend of increase compared with that in type II and negative groups, the difference among them is not significant. The inadequate number of participants maybe a factor attributing to this nonsignificant result. In future studies, we should further subdivide the pathological results based on severity of inflammation, and various precancerous changes from mild to severe. By analyzing histopathological results like that, a definite link between the different virulence of $H$. pylori strains and pathologic abnormalities may be established accurately.

There have been increasing documents showing that the prevalence of colorectal ailments has been linked to the

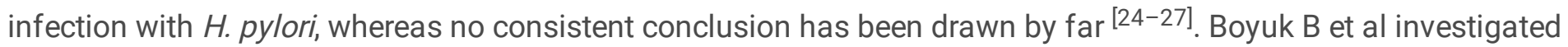
341 subjects and showed that the progression of colorectal adenoma and carcinoma is independent of $H$. pylori infection ${ }^{[24]}$. From our colonoscopic results, the incidences of colorectal polyps, and carcinoma with different virulent factors of $H$. pylori infection are not different among the three groups. However, the pathological diagnosis revealed that rates of severe inflammation, adenomatous polyp and colorectal cancer in patients with CagA/VacA toxins are much higher than that with UreA/B and negative group. Infection with $H$. pylori causes the secretion of gastrin in the blood, which can act as a hormone to stimulate the growth of the colonic mucosa cells. H. pylori can cause hypergastrinemia only or in combination with changes in the normal gastrointestinal flora, suggesting an acceptable mechanism for its carcinogenicity ${ }^{228,29]}$. The limitation of our study is the relatively small number of patients who received colonoscopy examinations. If we further group the colonoscopic findings according to size, location and morphology of intestinal lesions, the exact relationship could be defined.

\section{Conclusions}

In conclusion, the $\mathrm{H}$. Pylori strains carrying CagA/VacA are more likely to cause severe gastrointestinal diseases. However, the antibody measurement only reflects $H$. pylori infection, which does not differentiate whether it is due to previous infection or currently being infected status. Therefore, an accurate diagnosis of $H$. pylori infection should effectively be UBT and antibody tests simultaneously. Our findings further identify the exact effects of different virulence pathogenicity (CagA, VacA and UreA/B) on the development of gastrointestinal diseases. These may provide some clues and direction for the precise eradication of $H$. pylori in infected patients, and prevent gastrointestinal diseases in the future.

\section{Abbreviations}

H. pylori

Helicobacter Pylori

EGD

Esophagogastro duodenoscopy 
VacA

Vacuolating cytotoxin A

MALT

Mucosa-Associated Lymphoid Tissue lymphoma

UreA/B

Urease $A / B$

IARC

International Agency for Research on Cancer

WHO

World Health Organization

UBT

Urea breath test

\section{Declarations}

Ethics approval and consent to participate

The study was approved by the Ethical Committee of Affiliated Puren Hospital of Wuhan University of Science and Technology. All participants gave written informed consent.

Consent to publish

Not applicable.

Availability of data and materials

The datasets used and/or analyzed during the current study are available from the corresponding author on reasonable request.

Competing Interests

All authors declared no conflict of interest.

Author contributions

YZ, KT and XMF designed the experiments. KT and YZ summarized and wrote the draft. All authors read and approved the manuscript submission.

Funding

The authors would like to thank the Scientific Research Project of Wuhan Municipal Health Commission for research support to Y. Z. (Number: WX19Y09).

\section{References}

1. Sugano K, Tack J, Kuipers EJ, Graham DY, El-Omar EM, Miura S, Haruma K, Asaka M, Uemura N, Malfertheiner P; faculty members of Kyoto Global Consensus Conference. Kyoto global consensus report on Helicobacter pylori gastritis. Gut. 2015; 64(9):1353-67. PMID: 26187502 Doi: 10.1136/gutjnl-2015-309252. 
2. Malfertheiner P, Megraud F, O'Morain CA, Gisbert JP, Kuipers EJ, Axon AT, Bazzoli F, Gasbarrini A, Atherton J, Graham DY, Hunt R, Moayyedi P, Rokkas T, Rugge M, Selgrad M, Suerbaum S, Sugano K, El-Omar EM. European Helicobacter and Microbiota Study Group and Consensus panel. Management of Helicobacter pylori infectionthe Maastricht V/Florence Consensus Report. Gut. 2017;66(1):6-30. Doi:10.1136/gutjnl-2016-312288. PMID: 27707777.

3. Franceschi F, Covino M, Roubaud Baudron C, Review. Helicobacter pylori and extragastric diseases. Helicobacter. 2019;24(Suppl 1):e12636. Doi:10.1111/hel.12636. PMID: 31486239.

4. Yue F, Chuan Z, Shutian Z. The re-consideration of Helicobacter pylori infection. Chin J Dis. 2017;37(7):488-91. doi:10.3760/cma.j.issn.0254-1432.2017.07.015.

5. 2019 expert consensus opinion on eradication, prevention and control of gastric cancer in Helicobacter pylori China (Shanghai, 2019). Chin J Dis. 2019,39(5): 1-6.

6. Bakhti SZ, Latifi-Navid S, Safaralizadeh R. Helicobacter Pylori-Related Risk Predictors of Gastric Cancer: The Latest Models, Challenges, and Future Prospects. Cancer Med. 2020;9(13):4808-22. 10.1002/cam4.3068. PMID: 32363738 Doi:.

7. Nejati S, Karkhah A, Darvish H, Validi M, Ebrahimpour S, Nouri HR. Influence of Helicobacter pylori virulence factors CagA and VacA on pathogenesis of gastrointestinal disorders. Microb Pathog. 2018;117:43-8. Doi:10.1016/j.micpath.2018.02.016. PMID: 29432909.

8. Sukri A, Hanafiah A, Mohamad Zin N, Kosai NR. Epidemiology and role of Helicobacter pylori virulence factors in gastric cancer carcinogenesis. APMIS. 2020;128(2):150-61. Doi:10.1111/apm.13034. PMID: 32352605.

9. Ansari S, Yamaoka Y. Helicobacter pylori Virulence Factors Exploiting Gastric Colonization and its Pathogenicity. Toxins (Basel). 2019;11(11):677. Doi:10.3390/toxins11110677. PMID: 31752394.

10. Barden S, Lange S, Tegtmeyer N, Conradi J, Sewald N, Backert S, Niemann HH. A helical RGD motif promoting cell adhesion: crystal structures of the Helicobacter pylori type IV secretion system pilus protein CagL. Structure. 2013;21(11):1931-41. Doi:10.1016/j.str.2013.08.018. PMID: 24076404.

11. Canzian F, Rizzato C, Obazee O, Stein A, Flores-Luna L, Camorlinga-Ponce M, Mendez-Tenorio A, Vivas J, Trujillo E, Jang H, Chen W, Kasamatsu E, Bravo MM, Torres J, Muñoz N, Kato I. Genetic polymorphisms in the cag pathogenicity island of Helicobacter pylori and risk of stomach cancer and high-grade premalignant gastric lesions. Int J Cancer. 2020; PMID: 32363734 Doi: 10.1002/ijc.33032. Online ahead of print.

12. Chang WL, Yeh YC, Sheu BS. The impacts of H. pylori virulence factors on the development of gastroduodenal diseases. J Biomed Sci. 2018; 11;25(1):68. PMID: 30205817 Doi: 10.1186/s12929-018-0466-9.

13. Backert S, Blaser MJ. The Role of CagA in the Gastric Biology of Helicobacter pylori. Cancer Res. 2016;76(14):4028-31. Doi:10.1158/0008-5472.CAN-16-1680. PMID: 27655809.

14. Šterbenc A, Jarc E, Poljak M, Homan M. Helicobacter pylori virulence genes. World J Gastroenterol. 2019;25(33):4870-84. 10.3748/wjg.v25.i33.4870. PMID: 31543679 Doi:.

15. Whitmire JM, Merrell DS. Helicobacter pylori Genetic Polymorphisms in Gastric Disease Development. Adv Exp Med Biol. 2019;1149:173-94. PMID: 31016629 Doi: 10.1007/5584_2019_365.

16. McClain MS, Beckett AC, Cover TL. Helicobacter pylori Vacuolating Toxin and Gastric Cancer. Toxins (Basel). 2017;9(10):316. PMID: 29023421. Doi: 10.3390/toxins 9100316.

17. Ogiwara H, Graham DY, Yamaoka Y. VacA i-region subtyping. Gastroenterology. 2008;134(4):1267. 10.1053/j.gastro.2007.11.062. PMID: 18395110 Doi:. 
18. Basiri Z, Safaralizadeh R, Bonyadi MJ, Somi MH, Mahdavi M, Latifi-Navid S. Helicobacter pylori vacA d1 genotype predicts risk of gastric adenocarcinoma and peptic ulcers in northwestern Iran. Asian Pac J Cancer Prev. 2014;15(4):1575-9. Doi:10.7314/apjcp.2014.15.4.1575. PMID: 24641370.

19. Latifi-Navid S, Mohammadi S, Maleki P, Zahri S, Yazdanbod A, Siavoshi F, Massarrat S. Helicobacter pylori vacA d1/-i1 genotypes and geographic differentiation between high and low incidence areas of gastric cancer in Iran. Arch Iran Med. 2013;16(6):330-7. PMID: 23725065.

20. Letley DP, Rhead JL, Twells RJ, Dove B, Atherton JC. Determinants of non-toxicity in the gastric pathogen Helicobacter pylori. J Biol Chem. 2003;278(29):26734-41. 10.1074/jbc.M304071200. PMID: 12738773 Doi.

21. Amieva MR, El-Omar EM. Host-bacterial interactions in Helicobacter pylori infection. Gastroenterology. 2008;134(1):306-23. PMID: 18166359 Doi: 10.1053 /j.gastro.2007.11.009.

22. Raju D, Hussey S, Ang M, Terebiznik MR, Sibony M, Galindo-Mata E, Gupta V, Blanke SR, Delgado A, RomeroGallo J, Ramjeet MS, Mascarenhas H, Peek RM, Correa P, Streutker C, Hold G, Kunstmann E, Yoshimori T, Silverberg MS, Girardin SE, Philpott DJ, El Omar E, Jones NL. Vacuolating cytotoxin and variants in Atg16L1 that disrupt autophagy promote Helicobacter pylori infection in human. Gastroenterology. 2012;142(5):1160-71.

PMID: 22333951 Doi: 10.1053/ j. gastro. 2012.01.043.

23. Montecucco C, Rappuoli R. Living dangerously: how Helicobacter pylori survives in the human stomach. Nat Rev Mol Cell Biol. 2001;2(6):457-66. Doi:10.1038/35073084. PMID: 11389469.

24. Boyuk B, Ozgur A, Atalay H, Celebi A, Ekizoglu I, Aykurt E. Helicobacter pylori infection coexisting with intestinal metaplasia is not associated with colorectal neoplasms. Prz Gastroenterol. 2019;14(2):133-9. PMID: 31616528 Doi: $10.5114 /$ pg.2019.85897.

25. Kawahara Y, Kodama M, Mizukami K, Saito T, Hirashita Y, Sonoda A, Fukuda K, Matsunari O, Okamoto K, Ogawa R, Okimoto T, Murakami K. Endoscopic gastric mucosal atrophy as a predictor of colorectal polyps: a large scale case-control study. J Clin Biochem Nutr. 2019;65(2):153-9. Doi:10.3164/jcbn.19-47. PMID: 31592060.

26. Teimoorian F, Ranaei M, Hajian Tilaki K, Shokri Shirvani J, Vosough Z. Association of Helicobacter pylori Infection With Colon Cancer and Adenomatous Polyps. Iran J Pathol. 2018;13(3):325-32. PMID: 30636955.

27. Nam JH, Hong CW, Kim BC, Shin A, Ryu KH, Park BJ, Kim B, Sohn DK, Han KS, Kim J, Lee CW. Helicobacter pylori infection is an independent risk factor for colonic adenomatous neoplasms. Cancer Causes Control. 2017;28(2):107-15. Doi:10.1007/s10552-016-0839-x. PMID: 28025763.

28. Papastergiou V, Karatapanis S, Georgopoulos SD. Helicobacter pylori and colorectal neoplasia: Is there a causal link? World J Gastroenterol. 2016;22(2):649-58. Doi:10.3748/wjg.v22.i2.649. PMID: 26811614.

29. Georgopoulos SD, Polymeros D, Triantafyllou K, Spiliadi C, Mentis A, Karamanolis DG, Ladas SD. Hypergastrinemia is associated with increased risk of distal colon adenomas. Digestion 2006; 74: 42-46. PMID:17068397 doi: 10.1159/000096593.

\section{Figures}




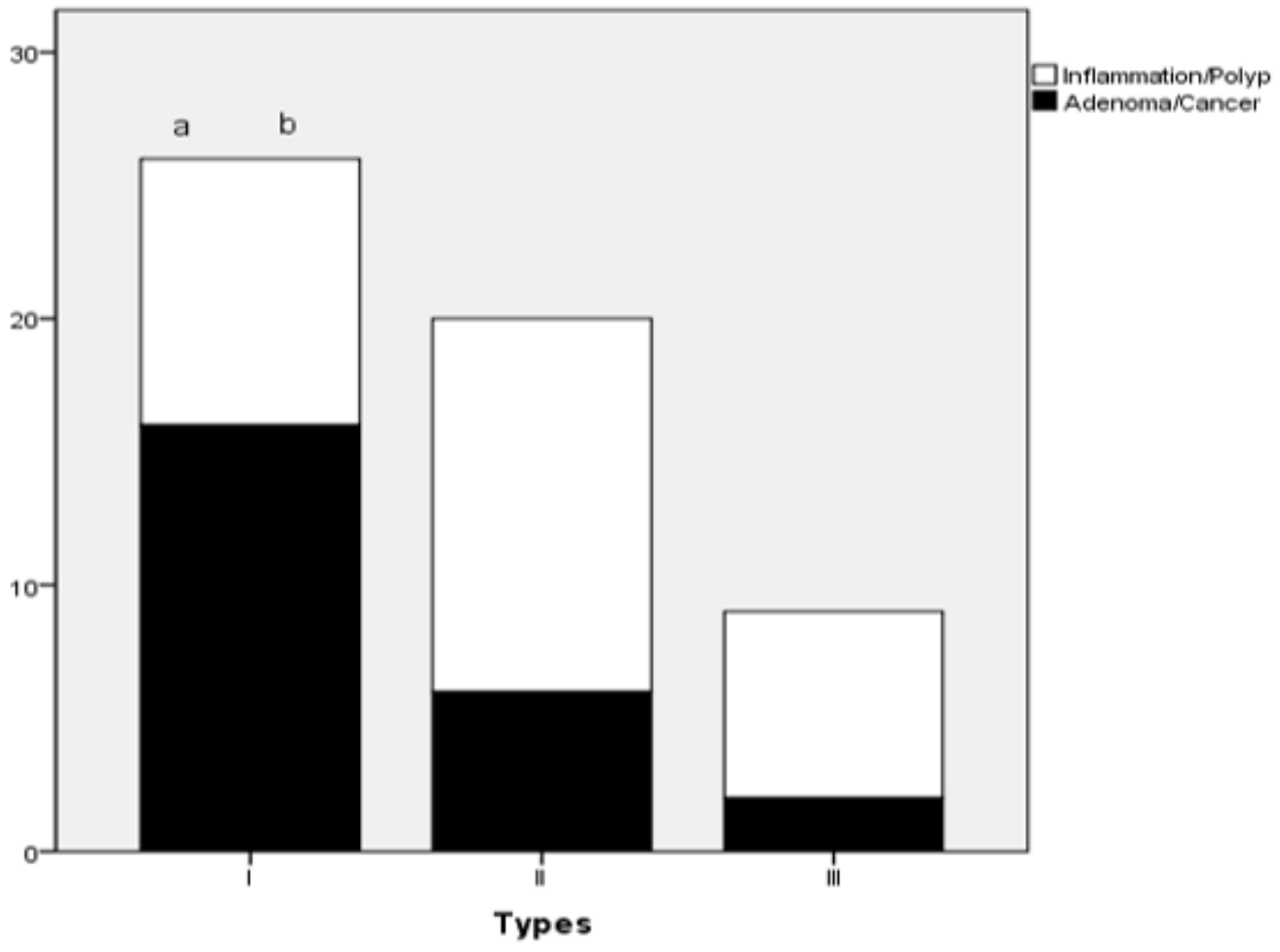

Figure 1

Pathological results of colonoscopy among different types of $\mathrm{H}$. pylori infection. a: $\mathrm{P}<0.05$, for comparing Type I and Type II groups. The incidences of severe inflammation, precancerous lesions and colorectal cancer were significantly increased. b:P®0.01 for comparing Type I and the negative groups. There was significant difference between the type I infection and negative groups. 